\title{
Optimum Number of Oocytes Retrieved Among Patients With Polycystic Ovary Syndrome Treated Using The Follicular Phase Long-Acting Long Protocol: A Retrospective Cohort Study
}

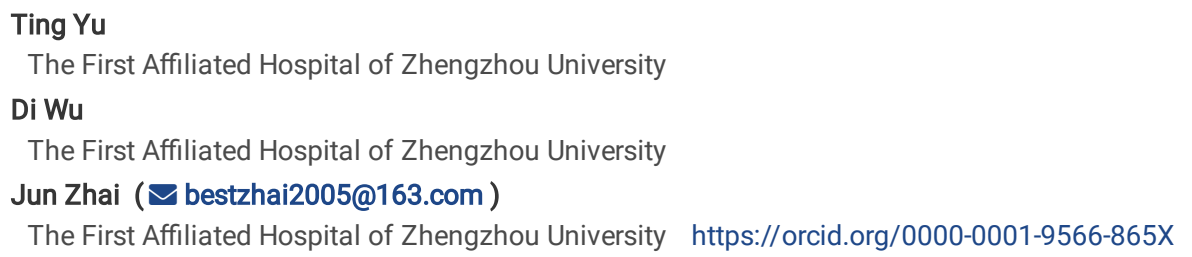

\section{Research}

Keywords: oocyte number, in vitro fertilization, intracytoplasmic sperm injection-embryo transfer, follicular phase long-acting long protocol, polycystic ovary syndrome

Posted Date: November 1st, 2021

DOI: https://doi.org/10.21203/rs.3.rs-997416/v1

License: (c) (1) This work is licensed under a Creative Commons Attribution 4.0 International License. Read Full License 


\section{Abstract}

Background: The optimum number of oocytes retrieved by the follicular phase long-acting long protocol is still unknown. This study aimed to analyze the optimum oocyte number in patients with polycystic ovary syndrome (PCOS) undergoing this protocol.

Methods: A total of 1816 PCOS patients aged <35 years who were undergoing their first cycle of in vitro fertilization or intracytoplasmic sperm injection (IVF/ICSI) between January 2017 and June 2019 were identified and reviewed. All patients underwent stimulation using a follicular phase long-acting long protocol. In this retrospective cohort study, patients were categorized into seven groups according to the number of oocytes retrieved (group A, 1-5; group $B$, 6-10; group $C, 11-15$; group $D, 16-20$; group $E, 21-25$; group $F, 26-30$; group $G,>30$ ). The main outcome indicators were the rates of high-quality embryo, fresh cycle pregnancy, cumulative pregnancy, and "freezing all" for high ovarian response. The cumulative pregnancy and "freezing all" rates for high ovarian response were analyzed using multivariate logistic analysis.

Results: The high-quality embryo rate decreased with the increase in the number of oocytes retrieved $(P<0.001)$. In the $<20$ oocyte group, the clinical and cumulative pregnancy rates increased with the number of oocytes retrieved, and the "freezing all" rate for high response was within $30 \%$. In the $>20$ oocyte group, with an increase in the number of oocytes retrieved, no significant change was found in the clinical and cumulative pregnancy rates ( $P>0.05)$; however, the incidence of "freezing all" rate for high response was significantly increased $(P<0.001)$. After correcting for confounding factors, the number of oocytes retrieved was an independent predictor of the "freezing all" rate for high ovarian response (odds ratio [OR], 1.085; 95\% confidence interval [Cl] 1.057-1.113) and cumulative pregnancy rate (OR 1.091, 95\% $\mathrm{Cl} 1.057-1.126)$. The high-quality embryo rate was significantly affected by the cumulative pregnancy rate (OR, 59.076; 95\% Cl: 29.591-117.938).

Conclusion: In PCOS patients aged $<35$ years treated using the follicular phase long-acting long protocol, considering clinical outcomes, laboratory indicators, and safety, appropriate ovarian stimulation should be used to control the number of oocytes retrieved at 11-20.

\section{Background}

In vitro fertilization (IVF)/intracytoplasmic sperm injection (ICSI) is a common treatment modality for infertile women with polycystic ovary syndrome (PCOS). The goal of controlled ovarian hyperstimulation $(\mathrm{COH})$ is to achieve higher clinical pregnancy rate and live birth rate while avoiding the incidence of moderateto-severe ovarian hyperstimulation syndrome (OHSS) [1]. Studies have shown that the number of oocytes retrieved can affect the number of embryos, which in turn affects the clinical pregnancy rate [2]. When the number of oocytes retrieved is too small, the number of high-quality embryos decreases, resulting in a decline in the clinical pregnancy rate and cumulative pregnancy rate. The retrieval of too many oocytes may reduce the clinical pregnancy rate because the hyperphysiological levels of hormones affect endometrial receptivity [3], and the number of oocytes retrieved simultaneously will significantly increase the risk of OHSS [4]. Therefore, the optimum number of oocytes can be used as an important indicator of an ideal pregnancy outcome and a reduction in the complications of IVF/ICSI. Studies have shown that the optimum number of oocytes differs for different populations and according to the protocol used for ovarian stimulation. For example, in a population with normal ovarian reserve, the optimal number of oocytes is approximately 15 using the long luteal phase gonadotropin-releasing hormone $(\mathrm{GnRH})$ agonist protocol [5-8], while for PCOS patients treated using the same protocol, the optimal number of oocytes is 10 [9]. The follicular phase long-acting long protocol is widely used in clinical practice because long-term GnRH agonist administration can improve endometrial receptivity and reduce the estradiol (E2) level on the days of human chorionic gonadotropin (hCG) injection, thereby improving the clinical pregnancy rate and lowering the OHSS rate. To the best of our knowledge, no relevant study has been conducted on the optimum number of oocytes among PCOS patients undergoing treatment using the follicular phase long-acting long protocol [5].

\section{Materials And Methods}

\section{Aim}

This retrospective cohort study aimed to comprehensively evaluate the laboratory and clinical outcomes of different oocytes retrieved from PCOS patients aged $<35$ years who underwent assisted pregnancy using the follicular phase long-acting long protocol and to discuss the optimum number of oocytes in this population.

\section{Patients}

The protocol of this retrospective cohort study was approved by the Ethical Review Committee of the First Affiliated Hospital of Zhengzhou University. Written informed consent was waived due to the retrospective nature of the study. The selected research participants were patients who underwent IVF/ICSI at the Reproductive Medicine Center of the First Affiliated Hospital of Zhengzhou University between January 2017 and June 2019. The clinical data of the patients were assessed in the first cycle of IVF/ICSI-assisted pregnancy.

All patients were younger than 35 years, diagnosed according to the Rotterdam criteria for PCOS, and undergoing their first IVF/ICSI cycle with the follicular phase long-acting long protocol. For the purposes of this study, patients with cycles involving oocyte donation, rescue ICSI, uterine abnormalities, and without retrieved oocytes were excluded from the analysis. Patients who had not gotten pregnant after 2 years but still had frozen embryos remaining were also excluded from the study. Therefore, the study included 1816 patients with one complete round of treatment with a fresh transfer cycle and subsequent frozen embryo transfer (ET) cycles.

In this study, patient characteristics, including age, body mass index (BMI), duration of infertility, type of infertility, anti-Müllerian hormone (AMH) levels, and follicle-stimulating hormone (FSH) levels, were evaluated. Data regarding other parameters, including gonadotropin dose, number of days of ovarian 
stimulation, number of oocytes retrieved, type of insemination, and fertilization rate, were also recorded. The primary outcome was the cumulative live birth rate.

\section{IVF/ICSI-ET}

Downregulation protocol: On the second to third days of menstruation, the patients were subcutaneously injected with a long-acting GnRH agonist (Diphereline, $3.75 \mathrm{mg}$; Beaufour Ipsen Industrie, Dreux, France). Thirty days later, blood samples were obtained from the patients to determine the serum levels of $\mathrm{FSH}$, luteinizing hormone (LH), E2, and progesterone. The sizes of the antral follicles were monitored via vaginal ultrasonography. The $\mathrm{COH}$ was initiated when the FSH level was $<5 \mathrm{IU} / \mathrm{L}$, the LH level was $<3 \mathrm{IU} / \mathrm{L}$, and the antral follicle was nearly $5 \mathrm{~mm}$ in diameter.

COH protocol: The individualized dosage of gonadotropin (GONAL-f; Merck Serono, Darmstadt, Germany) was determined based on the patient's age, AMH level, antral follicle count (AFC), BMI, and serum FSH level.

hCG injection and corpus luteum support standard: We administered $250 \mu \mathrm{g}$ of Azer (Merck Serono, Italy) and 2000 IU of hCG (Zhuhai Livzon Pharmaceutical) when the diameter of one dominant follicle was $\geq 20 \mathrm{~mm}$, the diameters of three follicles were $\geq 17 \mathrm{~mm}$, or two-thirds of the follicles had diameters $\geq 16 \mathrm{~mm}$. The eggs were harvested under vaginal ultrasound guidance $36-37 \mathrm{~h}$ after the injections were administered.

Fresh ET: The method of fertilization was based on semen quality. Fresh ET was performed 3-5 days after the oocyte retrieval based on embryo quality, endometrial status, and conditions of the patients. The transplant was cancelled if the patients were deemed to be at high risk for OHSS, the P level was $>3$ $\mathrm{ng} / \mathrm{mL}$, or a uterine effusion was demonstrated. Depending on the specific situation of each patient, different frozen-thawed ET protocols were formulated [14].

Frozen-thawed ET: Depending on the specific situation of each patient, different frozen-thawed ET protocols were formulated. These mainly included the following three methods: natural cycle, hormone replacement cycle, and downregulated hormone replacement cycle.

\section{Statistical analysis}

The patients were categorized into seven groups according to the number of oocytes retrieved: group $A, 1-5$; group $B, 6-10$; group $C, 11-15$; group $D, 16-20$; group E, 21-25; group F, 26-30; and group G, >30. Continuous variables were first assessed for normality of distribution using the Kolmogorov-Smirnov test. Data with normal distributions are presented as means with their standard deviations, and skewed data are presented as medians with their interquartile ranges. One-way analysis of variance or the Kruskal-Wallis test was used to compare multiple sets of measurement data, and the chi-squared test or Fisher's exact test was used to analyze multiple sets of count data, as appropriate. The results of count data are expressed as rates (\%). Between-group comparisons were performed using the chi-squared test, and comparisons among multiple groups were performed using the chi-squared segmentation method. Regarding treatment cycles, upon dividing the data into seven groups according to the number of oocytes retrieved, important aspects of the treatment outcomes were assessed, including the fresh cycle pregnancy rate, cumulative pregnancy rate, "freezing all" rate for high ovarian response, and high-quality embryos rate. Logistic regression analyses were performed to identify independent correlates of each possible confounding factor, particularly "freezing all" rate for high ovarian response and cumulative pregnancy outcomes after adjusting for other confounders identified in the univariate analysis. The number of oocytes retrieved was analyzed as a continuous variable. Linear regression analysis (stepwise regression) was used to screen independent related factors that may affect the number of oocytes retrieved, and the confounding factors identified in the single factor analysis were excluded. Regression coefficients ( $\beta$ values) and their $95 \%$ confidence intervals (Cls) were calculated. The size of the return effect was adjudged according to the absolute value of the standardized regression coefficient $(\beta)$. Statistical tests were performed using a two-tailed alpha value of 0.05 with Bonferroni corrections $(P<0.007$ after the correction was considered to indicate a difference between groups). The statistical analyses were performed using SPSS version 24.0 (IBM Corporation, Armonk, NY, USA).

\section{Results}

The data selection process with the number of cycles excluded (with reasons for exclusion) is shown in Figure 1. A total of 1816 cycles of young PCOS patients who underwent IVF/ICSI for the first cycle using the follicular phase long-acting long protocol were included with 16 cycles in group A, 203 cycles in group B, 480 cycles in group C, 485 cycles in group D, 309 cycles in group E, 182 cycles in group F, and 141 cycles in group G. Significant differences were found in the AMH, FSH, AFC, E2 on hCG injection day, LH, progesterone, and mean sizes of bilateral ovaries between the groups (all P<0.05) (Table 1 and Figure 2). 
Table 1

Comparison of patients' general characteristics and ovulation induction information.

\begin{tabular}{|c|c|c|c|c|c|c|c|}
\hline \multirow[t]{2}{*}{ Item } & \multicolumn{7}{|c|}{ Number of oocytes retrieved (n) } \\
\hline & $1-5$ & $6-10$ & $11-15$ & $16-20$ & $21-25$ & $26-30$ & $>i$ \\
\hline Cycle number & 16 & 203 & 480 & 485 & 309 & 182 & $1<$ \\
\hline Age (year) & $29.00(27.00,32.00)$ & $29.00(26.00,31.00)$ & $28.00(26.00,30.00)$ & $28.00(26.00,30.00)$ & $28.00(26.00,30.00)$ & $28.00(26.00,30.00)$ & $2 \varepsilon$ \\
\hline \multicolumn{8}{|l|}{$\begin{array}{l}\text { Type of } \\
\text { infertility }\end{array}$} \\
\hline $\begin{array}{l}\text { Primary } \\
\text { infertility (\%) }\end{array}$ & $75.00(12 / 16)$ & $66.50(135 / 203)$ & $71.88(345 / 480)$ & $66.39(322 / 485)$ & $66.34(205 / 309)$ & $64.84(118 / 182)$ & 64 \\
\hline $\begin{array}{l}\text { Secondary } \\
\text { infertility (\%) }\end{array}$ & $25.00(4 / 16)$ & $33.50(68 / 203)$ & $28.13(135 / 480)$ & $33.61(163 / 485)$ & $33.66(104 / 309)$ & $35.16(64 / 182)$ & 3 ! \\
\hline $\begin{array}{l}\text { Duration of } \\
\text { infertility } \\
\text { (year) }\end{array}$ & $3.50(1.25,5.75)$ & $3.00(2.00,5.00)$ & $3.00(2.00,5.00)$ & $3.00(2.00,5.00)$ & $3.00(2.00,5.00)$ & $3.00(2.00,5.00)$ & 3. \\
\hline $\mathrm{FSH}(\mathrm{mlU} / \mathrm{ml})$ & $6.74(6.21,7.41)^{\mathrm{DEF}}$ & $5.98(5.13,6.84)^{\mathrm{cde}}$ & $5.88(5.01,6.87)^{\delta}$ & $5.81(4.92,6.72)^{\square}$ & $5.58(4.79,6.36)^{\ddagger}$ & $5.53(4.76,6.36)$ & 5. \\
\hline $\mathrm{AMH}(\mathrm{ng} / \mathrm{ml})$ & $6.21(4.90,8.52)^{\mathrm{EF}}$ & $6.02(4.30,9.23)^{\mathrm{bcde}}$ & $6.84(5.14,9.62)^{\beta \gamma \delta}$ & $7.15(5.22,9.86)^{\square]}$ & $7.76(5.80,10.88)^{\ddagger}$ & $8.92(6.76,12)$ & $1 C$ \\
\hline BMI (kg/m2) & $25.40(21.85,27.28)$ & $24.50(21.85,26.70)$ & $23.80(21.40,26.30)$ & $24.40(21.93,26.90)$ & $24.70(22.20,27.10)$ & $24.40(22.2,26.75)$ & $2 !$ \\
\hline $\operatorname{AFC}(n)$ & $24(24,24)$ & $24(22,24)^{\mathrm{bcde}}$ & $24(23,24)^{Y}$ & $24(24,24)$ & $24(24,24)$ & $24(24,24)$ & 24 \\
\hline \multicolumn{8}{|l|}{$\begin{array}{l}\text { Fertilization } \\
\text { method }\end{array}$} \\
\hline IVF(\%) & $93.75(15 / 16)$ & 87.68(178/203) & $89.38(429 / 480)$ & $85.57(415 / 485)$ & 89.97(278/309) & $88.46(161 / 182)$ & $8 \varepsilon$ \\
\hline ICSI(\%) & $6.25(1 / 16)$ & $12.32(25 / 203)$ & $10.63(51 / 480)$ & $14.43(70 / 485)$ & 10.03(31/309) & $11.54(21 / 182)$ & 11 \\
\hline \multirow{2}{*}{$\begin{array}{l}\text { Starting dose } \\
\text { of } G n(I U)\end{array}$} & 112.50 & 112.50 & 112.50 & 112.50 & 112.50 & 100.00 & 11 \\
\hline & $(87.50,125.00)$ & $(100.00,118.75)$ & $(100.00,112.50)$ & $(100.00,112.50)$ & $(100.00,112.50)$ & $(100.00,112.50)$ & $(1$ \\
\hline $\begin{array}{l}\text { Length of } \\
\text { stimulation (d) }\end{array}$ & $15(13,19)$ & $14(12,16)^{\text {cde }}$ & $14(12,16)^{\beta \gamma \delta}$ & $14(13,16)$ & $15(13,17)$ & $15(13,17)$ & $1 !$ \\
\hline \multirow{2}{*}{$\begin{array}{l}\text { Total dosage } \\
\text { of Gn used (IU) }\end{array}$} & 2837.50 & 1937.50 & 1937.50 & 1925.00 & 2062.50 & 2118.75 & $2:$ \\
\hline & $(1687.50,4331.25)$ & $(1487.50,2687.50)$ & $(1468.75,2525.00)$ & $(1462.50,2537.50)$ & $(1500.00,2750.00)$ & $(1625.00,2653.13)$ & (1 \\
\hline \multicolumn{8}{|l|}{$\begin{array}{l}\text { hCG injection } \\
\text { day }\end{array}$} \\
\hline $\begin{array}{l}\text { Endometrial } \\
\text { thickness }(\mathrm{mm})\end{array}$ & $12.00(10.25,13.75)$ & $13.00(11.00,14.00)$ & $12.00(11.00,14.00)$ & $12.00(10.00,14.00)$ & $12.00(11.00,14.00)$ & $12.00(10.00,14.00)$ & $1 \%$ \\
\hline \multirow[t]{3}{*}{ E2 (pg/ml) } & 1113.00 & 2262.00 & 2906.00 & 3510.50 & 4350.00 & 5191.00 & $5 !$ \\
\hline & $(880.65$ & (1644.00, & (1979.50, & $(2625.50$ & (3286.00, & (3349.50, & (3 \\
\hline & $2423.00)^{\mathrm{BCDEF}}$ & $3074.00)^{B C D E F}$ & $4170.00)^{a \beta \gamma \delta}$ & $4861.50)^{\square 00}$ & $5587.00)^{\ddagger}$ & 6895.25) & $7:$ \\
\hline $\mathrm{P}(\mathrm{ng} / \mathrm{ml})$ & $0.27(0.09,0.54)^{\mathrm{CDEF}}$ & $0.48(0.29,0.71)^{\mathrm{abcde}}$ & $0.58(0.38,0.93)^{a \beta \gamma \delta}$ & $0.7(0.43,1.09)^{\square 00}$ & $0.84(0.57,1.28)^{\dagger \ddagger}$ & $1.01(0.65,1.53)$ & 1. \\
\hline LH (mlU/ml) & $0.75(0.17,1.81)$ & $0.57(0.28,0.90)^{\mathrm{ce}}$ & $0.52(0.25,0.86)^{\delta}$ & $0.50(0.25,0.80)^{\square}$ & $0.41(0.19,0.70)$ & $0.47(0.20,0.80)$ & 0. \\
\hline
\end{tabular}

Note:Continuous data: mean \pm SD. Categorical data: \% (n/N); BMI, body mass index; E2, estradiol; P, progesterone; FSH, follicle-stimulating hormone; AMH, Ant Antral follicular count; $\mathrm{Gn}$, gonadotropin; hCG, human chorionic gonadotropin.

${ }^{A} \mathrm{P},{ }^{\mathrm{B}} \mathrm{P},{ }^{\mathrm{C}} \mathrm{P},{ }^{\mathrm{D}} \mathrm{P}, \mathrm{E} \mathrm{P}$ and ${ }^{\mathrm{F} P}$ indicate group 1 vs. groups 2, 3, 4, 5, 6 and 7 respectively;

${ }^{\text {aP }},{ }^{\text {bP, }}{ }^{c} \mathrm{P},{ }^{d} \mathrm{P}$ and ${ }^{\mathrm{e}} \mathrm{P}$ indicate group 2 vs. groups $3,4,5,6$ and 7 respectively;

${ }^{a} P,{ }^{\beta} P, Y P$ and ${ }^{\delta} P$ indicate group 3 vs. groups 4, 5, 6 and 7 respectively;

${ }^{\square}, \rrbracket^{\square}$ and ${ }^{\natural} \mathrm{P}$ indicate group 4 vs. groups 5, 6 and 7 respectively;

${ }^{\dagger} \mathrm{P}$ and ${ }^{\ddagger} \mathrm{P}$ indicates group 5 vs.groups 6 and 7 respectively;

\#P indicates group 6 vs. group 7. 


\begin{tabular}{|c|c|c|c|c|c|c|c|}
\hline \multirow[t]{2}{*}{ Item } & \multicolumn{7}{|c|}{ Number of oocytes retrieved (n) } \\
\hline & $1-5$ & $6-10$ & $11-15$ & $16-20$ & $21-25$ & $26-30$ & $>i$ \\
\hline $\begin{array}{l}\text { Mean bilateral } \\
\text { ovarian } \\
\text { size }(\mathrm{mm})\end{array}$ & $4.35(3.93,4.90)^{\mathrm{CDEF}}$ & $4.85(4.10,5.50)^{\mathrm{bcde}}$ & $5.00(4.50,5.50)^{\mathrm{a} \beta \gamma \delta}$ & $5.30(4.85,5.90)^{\square[0]}$ & $5.55(5.00,6.20)^{\ddagger}$ & $5.80(5.10,6.51)$ & 6. \\
\hline \multicolumn{8}{|c|}{$\begin{array}{l}\text { Note:Continuous data: mean } \pm \text { SD. Categorical data: \% (n/N); BMI, body mass index; E2, estradiol; P, progesterone; FSH, follicle-stimulating hormone; AMH, Ant } \\
\text { Antral follicular count; Gn, gonadotropin; hCG, human chorionic gonadotropin. }\end{array}$} \\
\hline \multicolumn{8}{|c|}{${ }^{A} P,{ }^{B} P,{ }^{C} P,{ }^{D} P,{ }^{E} P$ and ${ }^{F} P$ indicate group 1 vs. groups $2,3,4,5,6$ and 7 respectively; } \\
\hline \multicolumn{8}{|c|}{${ }^{a} P,{ }^{b} P,{ }^{c} P,{ }^{d} P$ and ${ }^{e} P$ indicate group 2 vs. groups $3,4,5,6$ and 7 respectively; } \\
\hline \multicolumn{8}{|c|}{${ }^{a} P_{,}^{\beta} P_{,}^{\gamma} P$ and ${ }^{\delta} P$ indicate group 3 vs. groups $4,5,6$ and 7 respectively; } \\
\hline \multicolumn{8}{|c|}{${ }^{\square},{ }^{\rrbracket} P$ and ${ }^{\square} P$ indicate group 4 vs. groups 5,6 and 7 respectively; } \\
\hline \multicolumn{8}{|c|}{${ }^{\dagger} \mathrm{P}$ and ${ }^{\ddagger} \mathrm{P}$ indicates group 5 vs.groups 6 and 7 respectively; } \\
\hline${ }^{\# P}$ indicates gr & 6 vs. group 7. & & & & & & \\
\hline
\end{tabular}

Significant differences were also noted in the fertilization rate, high-quality embryo rate, and blastocyst formation rate (all $\mathrm{P}<0.001)$. With an increase in the number of oocytes retrieved, the fertilization rate was maintained at a high level for 11-20 oocytes (62.82-61.42\%) and then decreased, and the high-quality embryo rate was highest (67.09\%) when 6-10 oocytes were retrieved and then decreased. Significant differences were found in the "freezing all" rate for high ovarian response, transplanted blastocyst rate, and cumulative pregnancy rate $(P<0.001)$. With an increase in the number of oocytes retrieved, the "freezing all" rate for high ovarian response, transplanted blastocyst rate, and cumulative pregnancy rate increased significantly. The clinical pregnancy rate was not significantly different among the groups $(P=0.768)$, but remained at a high level when 11-20 oocytes were retrieved (78.77-80.13\%) (Table 2 and Figure 3 ). 
Table 2

Laboratory results and clinical outcomes.

\begin{tabular}{|c|c|c|c|c|c|c|c|}
\hline \multirow[t]{2}{*}{ Item } & \multicolumn{7}{|c|}{ Number of oocytes retrieved (n) } \\
\hline & $A: 1-5$ & B:6-10 & C:11-15 & D:16-20 & $\mathrm{E}: 21-25$ & $F: 26-30$ & G:: \\
\hline $\begin{array}{l}\text { Cycle } \\
\text { number }\end{array}$ & 16 & 203 & 480 & 485 & 309 & 182 & 14 \\
\hline \multicolumn{8}{|l|}{$\begin{array}{l}\text { Laboratory } \\
\text { outcomes }\end{array}$} \\
\hline $\begin{array}{l}\text { Tatol } \\
\text { number of } \\
\text { oocytes } \\
\text { retrieved(n) }\end{array}$ & 62 & 1742 & 6297 & 8689 & 7065 & 5050 & 50 \\
\hline $\begin{array}{l}\text { Average } \\
\text { number of } \\
\text { oocytes } \\
\text { retrieved(n) }\end{array}$ & $4(3.5,5)^{B C D E F}$ & $9(8,10)^{\text {abcde }}$ & $13(12,14)^{a \beta \gamma \delta}$ & $18(17,19)^{100}$ & $23(22,24)^{\dagger \neq}$ & $28(27,29)$ & 31 \\
\hline $\begin{array}{l}\text { fertilization } \\
\text { rate }(\%)\end{array}$ & $61.29(38 / 62)$ & $60.10(1047 / 1742)$ & $62.82(3956 / 6297)^{\beta ү \delta}$ & $61.42(5337 / 8689)^{\square}$ & $60.23(4255 / 7065)^{\ddagger}$ & $59.52(3006 / 5050)$ & 57 \\
\hline $\begin{array}{l}\text { high-quality } \\
\text { embryo } \\
\text { rate(\%) }\end{array}$ & $60.53(23 / 38)$ & $67.09(693 / 1033)^{\text {cde }}$ & $63.90(2503 / 3917)^{\mathrm{y} \delta}$ & $63.27(3336 / 5273)^{\boxplus}$ & $61.61(2595 / 4212)^{\ddagger}$ & $59.67(1768 / 2963)$ & 58 \\
\hline $\begin{array}{l}\text { Blastocysts } \\
\text { rate(\%) }\end{array}$ & $12.50(1 / 8)$ & $47.31(158 / 334)^{\mathrm{acd}}$ & $57.10(1130 / 1979)$ & $54.71(1783 / 3259)^{\square}$ & $58.30(1718 / 2947)^{\ddagger}$ & $56.20(1241 / 2208)$ & 53 \\
\hline \multicolumn{8}{|l|}{$\begin{array}{l}\text { clinical } \\
\text { outcomes }\end{array}$} \\
\hline $\begin{array}{l}\text { "freezing all” } \\
\text { for high } \\
\text { ovarian } \\
\text { response } \\
\text { rate(\%) }\end{array}$ & $0(0 / 16)^{D F}$ & $8.37(17 / 203)^{\text {bcde }}$ & $15.21(73 / 480)^{a \beta \gamma \delta}$ & $28.04(136 / 485)^{\square[010}$ & $39.81(123 / 309)^{\dagger \ddagger}$ & $63.38(119 / 482)^{\#}$ & 84 \\
\hline $\begin{array}{l}\text { Blastocysts } \\
\text { transfer } \\
\text { rate(\%) }\end{array}$ & $0(0 / 13)^{\mathrm{CDEF}}$ & $4.02(7 / 174)^{\mathrm{abcde}}$ & $19.18(75 / 391)^{a \beta \gamma \delta}$ & $47.02(142 / 302)^{0100}$ & $70.00(112 / 160)^{\dagger}$ & $85.71(42 / 49)$ & 94 \\
\hline $\begin{array}{l}\text { Clinical } \\
\text { pregnancy } \\
\text { rate(\%) }\end{array}$ & $69.23(9 / 13)$ & 77.01(134/174) & 78.77(308/391) & $80.13(242 / 302)$ & $78.13(125 / 160)$ & $77.56(38 / 49)$ & 66 \\
\hline $\begin{array}{l}\text { Embryo } \\
\text { implantation } \\
\text { rate(\%) }\end{array}$ & $40.00(10 / 25)$ & $57.50(184 / 320)$ & $56.24(347 / 617)$ & $56.41(176 / 312)$ & $58.95(56 / 95)$ & $57.14(8 / 14)$ & 10 \\
\hline $\begin{array}{l}\text { Blastocysts } \\
\text { implantation } \\
\text { rate(\%) }\end{array}$ & $0(0 / 0)$ & $100.00(7 / 7)$ & 77.33(58/75) & $83.10(118 / 142)$ & $75.89(85 / 112)$ & $76.19(32 / 42)$ & 64 \\
\hline $\begin{array}{l}\text { Abortion } \\
\text { rate(\%) }\end{array}$ & $0(0 / 13)$ & $9.20(16 / 174)$ & $9.97(39 / 391)$ & $7.95(24 / 302)$ & $5.63(9 / 160)$ & 18.37(9/49) & 11 \\
\hline $\begin{array}{l}\text { Live birth } \\
\text { rate(\%) }\end{array}$ & $69.23(9 / 13)$ & $67.24(117 / 174)$ & $67.77(265 / 391)$ & $70.20(212 / 302)$ & $70.00(112 / 160)$ & $59.18(29 / 49)$ & 55 \\
\hline $\begin{array}{l}\text { Cumulative } \\
\text { pregnancy } \\
\text { rate(\%) }\end{array}$ & $68.75(11 / 16)^{\mathrm{CDEF}}$ & $80.79(164 / 203)^{\text {abcde }}$ & $88.75(426 / 480)^{\beta \delta}$ & $91.34(443 / 485)$ & $95.15(294 / 309)$ & $95.05(173 / 182)$ & 96 \\
\hline
\end{tabular}

Note:Continuous data: mean \pm SD. Categorical data: $\%(n / N)$

${ }^{\mathrm{A} P},{ }^{\mathrm{B}} \mathrm{P},{ }^{\mathrm{C}} \mathrm{P},{ }^{\mathrm{D}} \mathrm{P},{ }^{\mathrm{E}} \mathrm{P}$ and ${ }^{\mathrm{F}} \mathrm{P}$ indicate group 1 vs. groups $2,3,4,5,6$ and 7 respectively;

${ }^{a} \mathrm{P},{ }^{b} \mathrm{P},{ }^{\mathrm{C}} \mathrm{P}, \mathrm{d}^{\mathrm{P}}$ and ${ }^{\mathrm{e}} \mathrm{P}$ indicate group 2 vs. groups $3,4,5,6$ and 7 respectively;

${ }^{a} \mathrm{P},{ }^{\beta} \mathrm{P}, \mathrm{YP}$ and ${ }^{\delta} \mathrm{P}$ indicate group 3 vs. groups 4, 5, 6 and 7 respectively;

${ }^{\square}, \rrbracket \mathrm{P}$ and ${ }^{\square} \mathrm{P}$ indicate group 4 vs. groups 5, 6 and 7 respectively;

${ }^{\dagger} \mathrm{P}$ and ${ }^{\ddagger} \mathrm{P}$ indicates group 5 vs.groups 6 and 7 respectively;

\#P indicates group 6 vs. group 7.

The "freezing all" for high ovarian response group had 587 cycles, and the ovarian normal-response group had 1229 cycles. To reduce the interference of confounding factors, a logistic regression analysis of the following variables was performed: age, type of infertility, duration of infertility, BMI, FSH, AMH, AFC, 
gonadotropin dose characteristics (starting dose, medication time, and total dose), characteristics on hCG injection day (endometrial thickness, E2, progesterone, LH, mean bilateral ovarian size), and number of oocytes retrieved. Statistically significant variables identified in the univariate analysis were included in the multivariate logistic regression analysis. The logistic regression analyses revealed that a woman's age (adjusted odds ratio [aOR] $0.932,95 \%$ $\mathrm{Cl} 0.893-0.972, \mathrm{P}<0.001), \log _{10}$ (total gonadotropin dose) (aOR=0.225, 95\% Cl 0.090-0.560, P=0.001), $\log _{10}$ (E2 level on hCG injection day) (aOR=5.520, 95\% $\mathrm{Cl} 2.784-10.946, \mathrm{P}<0.001)$, mean size of the bilateral ovaries on the $\mathrm{hCG}$ injection day (aOR=1.585, 95\% $\mathrm{Cl} 1.349-1.861, \mathrm{P}<0.001)$, and number of oocytes retrieved $(\mathrm{aOR}=1.132,95 \% \mathrm{Cl} 1.108-1.156, \mathrm{P}<0.001)$ were independent factors in the "freezing all" for high ovarian response group (Table 3$)$.

Table 3

Logistic regression analysis of "freezing all" for high ovarian response

\begin{tabular}{|c|c|c|c|c|c|c|}
\hline & $\begin{array}{l}\text { No "freezing all" } \\
(n=1229)\end{array}$ & $\begin{array}{l}\text { "freezing all" for high ovarian } \\
\text { response }(n=587)\end{array}$ & $\mathrm{OR}(95 \% \mathrm{Cl})$ & $\begin{array}{l}\mathrm{P} \\
\text { value }\end{array}$ & ${ }^{a} \mathrm{OR}(95 \% \mathrm{Cl})$ & $\begin{array}{l}\text { ap } \\
\text { value }\end{array}$ \\
\hline Age (year) & $28.41 \pm 3.13$ & $27.83 \pm 3.11$ & $0.942(0.913,0.972)$ & $<0.001$ & $0.932(0.894,0.972)$ & 0.001 \\
\hline Type of infertility & & & $0.829(0.67,1.026)$ & 0.085 & & \\
\hline Primary infertility (\%) & 66.31(815/1229) & $70.36(413 / 587)$ & & & & \\
\hline Secondary infertility (\%) & $33.69(414 / 1229)$ & $29.64(174 / 587)$ & & & & \\
\hline $\begin{array}{l}\text { Duration of infertility } \\
\text { (year) }\end{array}$ & $3.81 \pm 2.42$ & $3.54 \pm 2.12$ & $0.948(0.906,0.991)$ & 0.020 & $0.989(0.935,1.046)$ & 0.700 \\
\hline BMI (kg/m2) & $24.50 \pm 3.32$ & $23.87 \pm 3.35$ & $0.945(0.917,0.973)$ & $<0.001$ & $0.949(0.895,1.007)$ & 0.083 \\
\hline $\mathrm{FSH}(\mathrm{mlU} / \mathrm{ml})$ & $5.90 \pm 1.59$ & $5.62 \pm 1.54$ & $0.893(0.836,0.953)$ & 0.001 & $0.968(0.889,1.054)$ & 0.458 \\
\hline $\mathrm{AMH}(\mathrm{ng} / \mathrm{ml})$ & $7.77 \pm 3.90$ & $9.69 \pm 4.60$ & $1.111(1.085,1.137)$ & $<0.001$ & $1.020(0.986,1.054)$ & 0.249 \\
\hline $\mathrm{AFC}(\mathrm{n})$ & $22.19 \pm 4.78$ & $22.81 \pm 4.33$ & $1.032(1.008,1.057)$ & 0.009 & $1.000(0.970,1.030)$ & 0.993 \\
\hline \multicolumn{7}{|l|}{ Gn } \\
\hline Starting dose of $\mathrm{Gn}(\mathrm{IU})$ & $109.05 \pm 19.42$ & $103.80 \pm 15.33$ & $0.982(0.976,0.988)$ & $<0.001$ & $1.002(0.990,1.013)$ & 0.770 \\
\hline Length of stimulation (d) & $14.59 \pm 2.65$ & $14.6 \pm 2.62$ & $1.001(0.965,1.039)$ & 0.948 & & \\
\hline $\begin{array}{l}\text { Log10(Total dosage of } \\
\text { Gn used) (IU) }\end{array}$ & $3.31 \pm 0.17$ & $3.27 \pm 0.17$ & $0.227(0.126,0.41)$ & $<0.001$ & $0.225(0.090,0.560)$ & 0.001 \\
\hline \multicolumn{7}{|l|}{ hCG injection day } \\
\hline $\begin{array}{l}\text { Endometrial } \\
\text { thickness(mm) }\end{array}$ & $12.38 \pm 2.36$ & $12.17 \pm 2.43$ & $0.964(0.925,1.005)$ & 0.085 & & \\
\hline $\log 10(\mathrm{E} 2)(\mathrm{pg} / \mathrm{ml})$ & $3.48 \pm 0.23$ & $3.66 \pm 0.21$ & $41.707(24.793,70.16)$ & $<0.001$ & $5.520(2.784,10.946)$ & $<0.001$ \\
\hline $\mathrm{P}(\mathrm{ng} / \mathrm{ml})$ & $0.77 \pm 0.51$ & $0.97 \pm 0.58$ & $1.005(0.902,1.119)$ & 0.927 & & \\
\hline $\mathrm{LH}(\mathrm{mlU} / \mathrm{ml})$ & $0.71 \pm 0.91$ & $0.71 \pm 0.93$ & $1.962(1.636,2.353)$ & $<0.001$ & $0.982(0.847,1.140)$ & 0.815 \\
\hline $\begin{array}{l}\text { Mean bilateral ovarian } \\
\text { size }(\mathrm{mm})\end{array}$ & $5.14 \pm 0.86$ & $5.90 \pm 1.01$ & $2.477(2.188,2.804)$ & $<0.001$ & $1.585(1.349,1.861)$ & $<0.001$ \\
\hline $\begin{array}{l}\text { Number of oocytes } \\
\text { retrieved(n) }\end{array}$ & $16.20 \pm 5.95$ & $23.90 \pm 8.35$ & $1.168(1.148,1.189)$ & $<0.001$ & $1.132(1.108,1.156)$ & $<0.001$ \\
\hline
\end{tabular}

Note:Continuous data: mean $\pm S D$. Categorical data: $\%(n / N) ;{ }^{a} O R,{ }^{a} P$, adjusted by the variables which were statistically significant in the univariate logistic analysis.

The cumulative pregnancy group included 1648 cycles, and 168 cycles did not reach pregnancy. The factors and methods included in the analysis were the same as those used in the logistic regression analysis of "freezing all" for high ovarian response. The results showed that log ${ }_{10}$ (E2 level on hCG injection day), number of oocytes retrieved, and high-quality embryo rate were factors that influenced the cumulative pregnancy rate $(P<0.05)$. The multivariate logistic regression analysis showed that the number of oocytes retrieved (aOR=1.091, 95\% $\mathrm{Cl} 1.057-1.126, \mathrm{P}<0.001)$ and high-quality embryo rate $(\mathrm{aOR}=59.076,95 \%$ $\mathrm{Cl} 29.591-117.938, \mathrm{P}<0.001)$ were independent predictors of cumulative pregnancy (Tables 4 and 5 ). 
Table 4

Single factor logistic regression analysis of cumulative pregnancy

\begin{tabular}{|c|c|c|c|c|}
\hline & $\begin{array}{l}\text { No cumulative pregnancy } \\
(n=168)\end{array}$ & $\begin{array}{l}\text { Cumulative pregnancy } \\
(n=1648)\end{array}$ & $\mathrm{OR}(95 \% \mathrm{Cl})$ & $P$ value \\
\hline Age (year) & $28.26 \pm 3.23$ & $28.22 \pm 3.13$ & $0.996(0.947,1.048)$ & 0.875 \\
\hline Type of infertility & & & $1.181(0.834,1.674)$ & 0.348 \\
\hline Primary infertility (\%) & $119 / 168$ & $1109 / 1648$ & & \\
\hline Secondary infertility (\%) & $49 / 168$ & $539 / 1648$ & & \\
\hline Duration of infertility (year) & $4.01 \pm 2.48$ & $3.69 \pm 2.31$ & $0.948(0.890,1.010)$ & 0.098 \\
\hline BMI (kg/m2) & $24.30 \pm 3.38$ & $24.30 \pm 3.34$ & $1.000(0.954,1.049)$ & 1.000 \\
\hline $\mathrm{FSH}(\mathrm{mlU} / \mathrm{ml})$ & $5.93 \pm 1.78$ & $5.80 \pm 1.56$ & $0.95(0.860,1.049)$ & 0.309 \\
\hline $\mathrm{AMH}(\mathrm{ng} / \mathrm{ml})$ & $8.19 \pm 4.15$ & $8.41 \pm 4.25$ & $1.013(0.974,1.052)$ & 0.527 \\
\hline AFC (n) & $22.34 \pm 4.25$ & $22.40 \pm 4.69$ & $1.003(0.969,1.037)$ & 0.880 \\
\hline \multicolumn{5}{|l|}{$\mathrm{Gn}$} \\
\hline Starting dose of Gn(IU) & $108.41 \pm 22.04$ & $107.25 \pm 17.95$ & $0.997(0.989,1.005)$ & 0.435 \\
\hline Length of stimulation (d) & $14.74 \pm 2.52$ & $14.57 \pm 2.66$ & $0.976(0.92,1.036)$ & 0.429 \\
\hline Log10(Total dosage of Gn used) (IU) & $3.31 \pm 0.16$ & $3.29 \pm 0.17$ & $0.49(0.191,1.255)$ & 0.137 \\
\hline \multicolumn{5}{|l|}{ hCG injection day } \\
\hline Endometrial thickness(mm) & $12.49 \pm 2.62$ & $12.30 \pm 2.36$ & $0.966(0.905,1.033)$ & 0.312 \\
\hline $\log 10(\mathrm{E} 2)(\mathrm{pg} / \mathrm{ml})$ & $3.49 \pm 0.26$ & $3.55 \pm 0.23$ & $2.629(1.352,5.111)$ & 0.004 \\
\hline $\mathrm{P}(\mathrm{ng} / \mathrm{ml})$ & $0.80 \pm 0.53$ & $0.84 \pm 0.54$ & $1.121(0.828,1.518)$ & 0.459 \\
\hline $\mathrm{LH}(\mathrm{mlU} / \mathrm{ml})$ & $0.67 \pm 0.80$ & $0.72 \pm 0.92$ & $1.077(0.884,1.313)$ & 0.460 \\
\hline Mean bilateral ovarian size(mm) & $5.33 \pm 0.98$ & $5.39 \pm 0.98$ & $1.065(0.904,1.255)$ & 0.452 \\
\hline Number of oocytes retrieved(n) & $15.20 \pm 6.91$ & $19.05 \pm 7.70$ & $1.085(1.057,1.113)$ & $<0.001$ \\
\hline high-quality embryo rate(\%) & $0.37 \pm 0.31$ & $0.65 \pm 0.25$ & $57.494(28.797,114.786)$ & $<0.001$ \\
\hline
\end{tabular}

Table 5

Multivariate logistic regression analysis of cumulative pregnancy

\begin{tabular}{|lllll|}
\hline & \multicolumn{2}{l}{ Single factor logistic regression analysis } & \multicolumn{2}{l|}{ Multivariate logistic regression analysis } \\
\cline { 2 - 5 } & OR(95\%Cl) & P value & aOR(95\%Cl) & aP value \\
\hline $\log _{10}(\mathrm{hCG}$ injection day E2) $(\mathrm{pg} / \mathrm{ml})$ & $2.629(1.352,5.111)$ & 0.004 & $1.048(0.459,2.395)$ & 0.911 \\
\hline Number of oocytes retrieved $(\mathrm{n})$ & $1.085(1.057,1.113)$ & $<0.001$ & $1.091(1.057,1.126)$ & $<0.001$ \\
\hline high-quality embryo rate(\%) & $57.494(28.797,114.786)$ & $<0.001$ & $59.076(29.591,117.938)$ & $<0.001$ \\
\hline
\end{tabular}

The patient's age, duration of infertility, AMH level, FSH level, AFC, gonadotropin characteristics (starting dose, medication time, and total dose), and other variables as alternative independent variables and the number of oocytes retrieved as a dependent variable were entered into the multiple regression equation. The independent variables identified in the regression and order of the size of the influence on the number of eggs obtained were FSH ( $\beta=-0.731, P<0.001)$, duration of gonadotropin administration $(\beta=0.374, P<0.001)$, AMH level $(\beta=0.322, P<0.001)$, and AFC $(\beta=0.085, P=0.035)($ Table 6$)$. 
Table 6

Factors that affect the number of oocytes retrieved

\begin{tabular}{|c|c|c|c|c|c|c|}
\hline Model & $\begin{array}{l}\text { Regression coefficients } \\
\text { b }\end{array}$ & $\begin{array}{l}\text { Standard error } \\
\text { Sx }\end{array}$ & $\begin{array}{l}\text { Standardized regression coefficient } \\
\text { B }\end{array}$ & $\mathrm{T}$ value & Sig. & $95.0 \% \mathrm{Cl}$ for $\mathrm{b}$ \\
\hline constant & 12.828 & 1.401 & & 9.159 & $<0.001$ & $12.828-1.401$ \\
\hline $\mathrm{AMH}(\mathrm{ng} / \mathrm{ml})$ & 0.322 & 0.044 & 0.177 & 7.340 & $<0.001$ & $0.322-0.044$ \\
\hline FSH (mIU/ml) & -0.731 & 0.116 & -0.151 & -6.279 & $<0.001$ & $-0.731-0.116$ \\
\hline Length of stimulation (d) & 0.374 & 0.071 & 0.128 & 5.297 & $<0.001$ & $0.374-0.071$ \\
\hline $\operatorname{AFC}(n)$ & 0.085 & 0.040 & 0.050 & 2.108 & 0.035 & $0.085-0.040$ \\
\hline
\end{tabular}

\section{Discussion}

In this study, we determined the optimum number of oocytes for PCOS patients aged < 35 years. Considering the clinical outcomes, laboratory indicators, and safety, the optimum number of oocytes was 11-20. Studies have shown differences in the optimum number of oocytes between different ovarian response groups and patients treated using different ovarian stimulation protocols [16-17]. In the population with a normal ovarian response, the GnRH antagonist protocol has the best effect in obtaining 10-11 oocytes, while the GnRH agonist protocol can achieve the highest clinical pregnancy rate when 8-11 oocytes are obtained [18]. After comprehensively evaluating the cumulative live birth rate and remaining embryo rate of PCOS patients who underwent assisted pregnancy using the luteal phase long GnRH agonist protocol, a retrospective study suggested that the optimum number of oocytes was 10 [9]. Because PCOS patients with high ovarian responsiveness have a large number of antral follicles, their AMH levels are higher than those of normal people [19]. During the COH process, the number of oocytes retrieved is often large; however, the quality is poor, resulting in a low fertilization rate and a higher risk of OHSS [20]. Therefore, the optimum number of oocytes for women with normal ovarian response may not apply to PCOS patients. Since the follicular phase long-acting long protocol uses a lower dose than the luteal phase long GnRH agonist protocol, the E2 level on the day of hCG administration is lower and the long-acting $\mathrm{GnRH}$ agonist improves endometrial receptivity. This can increase the fresh cycle transplantation rate and clinical pregnancy rate, and reduce the risk of OHSS $(P<0.01)[12,21]$. In China, the follicular phase long-acting long protocol has become one of the predominant $\mathrm{COH}$ protocols used in the field of assisted reproduction. To reduce the risk of OHSS, GnRH antagonist protocols are often used to assist pregnancy among PCOS patients. Current research shows no significant difference in the OHSS incidence between patients treated using the follicular phase long-acting long protocol and those treated using the GnRH antagonist protocol [10-11]. Therefore, it is of great clinical significance to study the optimum number of oocytes among PCOS patients treated using the follicular phase long-acting long protocol.

As the strategy of transplanting fresh embryos is adopted in the follicular phase long-acting long protocol, it is particularly important to obtain an appropriate number of oocytes. In this study, laboratory indicators were combined with clinical results to determine the optimum number of oocytes. The results showed that the high-quality embryo rate decreased gradually with an increase in the number of oocytes retrieved. When $<20$ oocytes were retrieved, the fertilization rate was higher, and this rate decreased with an increase in oocytes retrieved when $>20$ were retrieved $(P<0.007)$. No significant difference was found in the clinical pregnancy rate among the groups; however, the clinical pregnancy rate was the highest (80.13\%) when 16-20 oocytes were retrieved. Additionally, the excessive number of oocytes retrieved may be related to adverse pregnancy outcomes. A study involving 65,868 single live births showed that $>20$ oocytes were retrieved in assisted reproductive technology, which may be related to the increased risk of premature birth and low birth weight among infants [22]. The cumulative pregnancy rate/live birth rate has become a more meaningful index for evaluating IVF-ET [23]. A systematic retrospective analysis showed that the retrieval of 12-18 oocytes can yield the maximum fresh cycle live birth rate and a continuous positive correlation exists between the number of oocytes retrieved and the cumulative live birth rate [24]. A study involving 39,387 individuals treated using the $\mathrm{GnRH}$ agonist or GnRH antagonist protocol, including 77,956 fresh cycles and 36,270 frozen cycles, suggested that the retrieval of 18-20 oocytes can achieve the desired cumulative live birth rate and maintain the incidence of OHSS at a reasonable level [25]. Another study including 221,221 treatment cycles showed that women aged 18-34 years achieved the highest cumulative live birth rate when 25 oocytes were retrieved and that with the continuous increase in the number of oocytes retrieved, the cumulative live birth rate increased a little.

The multiple logistic regression analysis showed that the number of oocytes retrieved and the high-quality embryo rate were independent risk factors for cumulative pregnancy. When $<20$ oocytes were retrieved, the cumulative pregnancy rate increased significantly with an increase in the number of oocytes retrieved $(P<0.007)$. When $>20$ oocytes were retrieved, the cumulative pregnancy rate did not increase. According to the comprehensive clinical and laboratory results, when 11-20 oocytes were retrieved, the high-quality embryo rate was high, the "freezing all" for high ovarian response rate was controlled within an acceptable range, and fresh cycle pregnancy and cumulative pregnancy rates both reached peak values.

To our knowledge, this study is the first to analyze the optimal number of oocytes among PCOS patients undergoing their first IVF/ICSI cycle with the follicular phase long-acting long protocol by considering clinical outcomes, laboratory indicators, and adverse outcomes, such as "freezing all" for high ovarian response. This study provides a basis for assisted conception management of PCOS patients in clinical practice via IVF/ICSI-ET. Simultaneously, we realize that our study had some limitations. First this study utilized a retrospective design without considering all confounding factors. Second, the study only included PCOS patients aged < 35 years. Third, the conclusions derived herein cannot be extended to all age groups or patients with low or normal ovarian reserves. Meanwhile, the cut-off point of the observation time was 2 years, and some patients had frozen embryos that had not been transplanted. These 
members of the population were excluded from this study because the number of patients was small (69 cycles in total). The results of the present study need to be further confirmed in large, multicenter, and prospective studies.

\section{Conclusion}

This study comprehensively analyzed the clinical pregnancy rate, cumulative pregnancy rate, high-quality embryo rate, and "freezing all" rate for high ovarian response. Among young PCOS patients undergoing assisted pregnancy using the follicular phase long-acting long protocol, an optimum number of $11-20$ oocytes is required to retrieved better clinical pregnancy outcomes and a low risk of OHSS.

\section{Abbreviations}

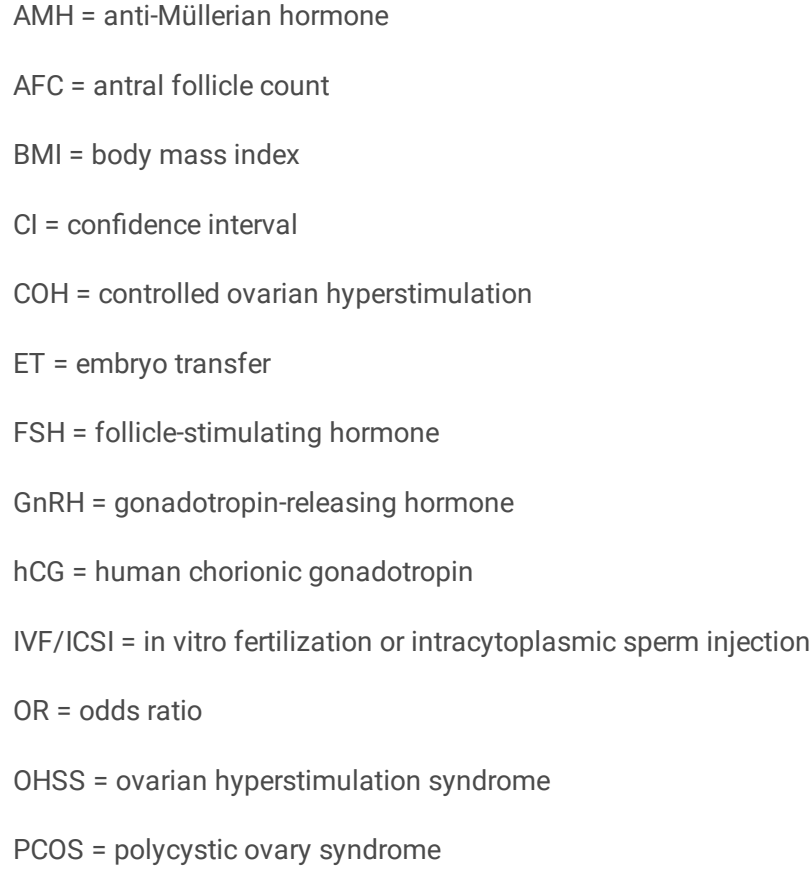

\section{Declarations}

Ethics approval and consent to participate: The protocol of this retrospective cohort study was approved by the Ethical Review Committee of the First Affiliated Hospital of Zhengzhou University. Written informed consent was waived due to the retrospective nature of the study.

Consent for publication:Not applicable.

Availability of data and materials :The datasets that were used and/or analysed during the current study are available from the corresponding author upon reasonable request.

Competing interests: Not applicable

Funding: This study was supported by the National Natural Science Foundation of China (grant no. 82071649).

Authors' contributions: JZ contributed to the conception of this study. TY was responsible for the study design, statistical analyses, and manuscript writing. DW contributed by revising the manuscript. All the authors contributed to the manuscript and approved the submitted version.

\section{Acknowledgements}

We thank all medical staff and patients of the First Affiliated Hospital of Zhengzhou University for recording the data and cooperating with treatments, respectively.

\section{References}

1. Oudshoorn SC. van Tilborg TC, Eijkemans MJC, Oosterhuis GJE. Friederich J, van Hooff MHA, van Santbrink EJP, Brinkhuis EA. Smeenk JMJ, Kwee J, et al. Individualized versus standard FSH dosing in women starting IVF/ICSI: an RCT. Part 2: The predicted hyper responder. Hum Reprod. 2017 Dec 1;32(12):2506-2514.

2. Swain JE. Pool TB. ART failure: oocyte contributions to unsuccessful fertilization. Hum Reprod Update. 2008 Sep-Oct,14(5):431-46.

3. Evans J. Hannan NJ, Edgell TA. Vollenhoven BJ, Lutjen PJ. Osianlis T, Salamonsen LA. Rombauts LJ. Fresh versus frozen embryo transfer: backing clinical decisions with scientific and clinical evidence. Hum Reprod Update. 2014 Nov-Dec,20(6):808-21. 
4. Steward RG. Lan L, Shah AA. Yeh JS, Price TM. Goldfarb JM, Muasher SJ. Oocyte number as a predictor for ovarian hyperstimulation syndrome and live birth: an analysis of 256,381 in vitro fertilization cycles. Fertil Steril. 2014 Apr;101(4):967-73.

5. Ji J. Liu Y, Tong XH. Luo L, Ma J. Chen Z. The optimum number of oocytes in IVF treatment: an analysis of 2455 cycles in China. Hum Reprod. 2013 Oct,28(10):2728-34.

6. Zhou J. Wang B, Hu Y. Sun H. Association between the number of oocytes retrieved and cumulative live birth rate in women aged 35-40 years undergoing long GnRH agonist IVF/ICSI cycles. Arch Gynecol Obstet. 2017 Nov,296(5):1005-12.

7. Sunkara SK. Rittenberg V, Raine-Fenning N. Bhattacharya S, Zamora J. Coomarasamy A. Association between the number of eggs and live birth in IVF treatment: an analysis of 400135 treatment cycles. Hum Reprod. 2011 Jul,26(7):1768-74.

8. Brodin T. Hadziosmanovic N, Berglund L. Olovsson M, Holte J. Comparing four ovarian reserve markers--associations with ovarian response and live births after assisted reproduction. Acta Obstet Gynecol Scand. 2015 Oct,94(10):1056-63.

9. Chen YH. Wang Q, Zhang YN. Han X, Li DH. Zhang CL. Cumulative live birth and surplus embryo incidence after frozen-thaw cycles in PCOS: how many oocytes do we need? J Assist Reprod Genet. 2017 Sep,34(9):1153-9.

10. Chen Y. Zhao J, Zhang H. Comparative Effectiveness of Three Ovarian Hyperstimulation Protocol in In Vitro Fertilization (IVF) Cycles for Women with Polycystic Ovary Syndrome. Med Sci Monit. 2018 Dec 28;24:9424-8.

11. Griesinger G. Diedrich K, Tarlatzis BC. Kolibianakis EM. GnRH-antagonists in ovarian stimulation for IVF in patients with poor response to gonadotrophins, polycystic ovary syndrome, and risk of ovarian hyperstimulation: a meta-analysis. Reprod Biomed Online. 2006 Nov,13(5):628-38.

12. Wu L. Ren XL, Chen W. Huang B, Zhou YF. Jin L. Influence of Different Gonadotropin-releasing Hormone Agonist Administration Methods on Pregnancy Outcomes of Patients Undergoing In-vitro Fertilization-embryo Transfer. Curr Med Sci. 2019 Jun,39(3):437-41.

13. Malchau SS. Henningsen AA, Forman J. Loft A, Nyboe Andersen A. Pinborg A. Cumulative live birth rate prognosis based on the number of aspirated oocytes in previous ART cycles. Hum Reprod. 2019 Jan 1;34(1):171-180..

14. Cao Y. Shi H, Ma Y. Ma L, Zhai J. Effect and Relationship of Seasons on the High Risk of Ovarian Hyperstimulation Syndrome After Oocyte Retrieval in Patients With Polycystic Ovary Syndrome. Front Endocrinol (Lausanne). 2021 Jan 26;11:610828.

15. Yue M. Ma L, Cao Y. Zhai J. Application of a Nomogram for Predicting the Risk of Subchorionic Hematoma in Early Pregnancy With In Vitro FertilizationEmbryo Transfer/Frozen Embryo Transfer. Front Endocrinol (Lausanne). 2021 Mar 2;12:631097.

16. Toftager M. Bogstad J, Løssl K. Prætorius L, Zedeler A. Bryndorf T, Nilas L. Pinborg A. Cumulative live birth rates after one ART cycle including all subsequent frozen-thaw cycles in 1050 women: secondary outcome of an RCT comparing GnRH-antagonist and GnRH-agonist protocols. Hum Reprod. 2017 Mar 1;32(3):556-567..

17. Verberg MF. Eijkemans MJ, Macklon NS. Heijnen EM, Baart EB. Hohmann FP, Fauser BC. Broekmans FJ. The clinical significance of the retrieval of a low number of oocytes following mild ovarian stimulation for IVF: a meta-analysis. Hum Reprod Update. 2009 Jan-Feb,15(1):5-12.

18. Kim H. Han SJ, Hong YS. Kim SW, Ku SY. Suh CS, Kim SH. Optimal Oocyte Number in Controlled Ovarian Stimulation with Gonadotropin-Releasing Hormone Agonist/Antagonist and Day 3 Fresh Embryo Transfer. Reprod Sci. 2021 Mar 24.

19. Practice Committee of the American Society for Reproductive Medicine. Electronic address: ASRM@asrm.org; Practice Committee of the American Society for Reproductive Medicine. Prevention and treatment of moderate and severe ovarian hyperstimulation syndrome: a guideline. Fertil Steril. 2016 Dec;106(7):1634-47.

20. Qiao J. Feng HL. Extra- and intra-ovarian factors in polycystic ovary syndrome: impact on oocyte maturation and embryo developmental competence. Hum Reprod Update. 2011 Jan-Feb,17(1):17-33.

21. Gong F. Li X, Zhang S. Ma H, Cai S. Li J, Lin GE. Lu G. A modified ultra-long pituitary downregulation protocol improved endometrial receptivity and clinical outcome for infertile patients with polycystic ovarian syndrome. Exp Ther Med. 2015 Nov,10(5):1865-70.

22. Sunkara SK. La Marca A, Seed PT, Khalaf Y. Increased risk of preterm birth and low birthweight with very high number of oocytes following IVF: an analysis of 65868 singleton live birth outcomes. Hum Reprod. 2015 Jun,30(6):1473-80.

23. Chambers GM. Paul RC, Harris K. Fitzgerald O, Boothroyd CV. Rombauts L, Chapman MG. Jorm L. Assisted reproductive technology in Australia and New Zealand: cumulative live birth rates as measures of success. Med J Aust. 2017 Aug 7;207(3):114-118..

24. Law YJ. Zhang N, Kolibianakis EM. Costello MF, Keller E. Chambers GM, Venetis CA. Is there an optimal number of oocytes retrieved at which live birth rates or cumulative live birth rates per aspiration are maximized after ART? A systematic review. Reprod Biomed Online. 2021 Jan;42(1):83-104.

25. Magnusson Å. Källen K, Thurin-Kjellberg A. Bergh C. The number of oocytes retrieved during IVF: a balance between efficacy and safety. Hum Reprod. 2018 Jan 1;33(1):58-64..

26. Law YJ. Zhang N, Venetis CA. Chambers GM, Harris K. The number of oocytes associated with maximum cumulative live birth rates per aspiration depends on female age: a population study of 221221 treatment cycles. Hum Reprod. 2019 Sep 29;34(9):1778-87.

\section{Figures}




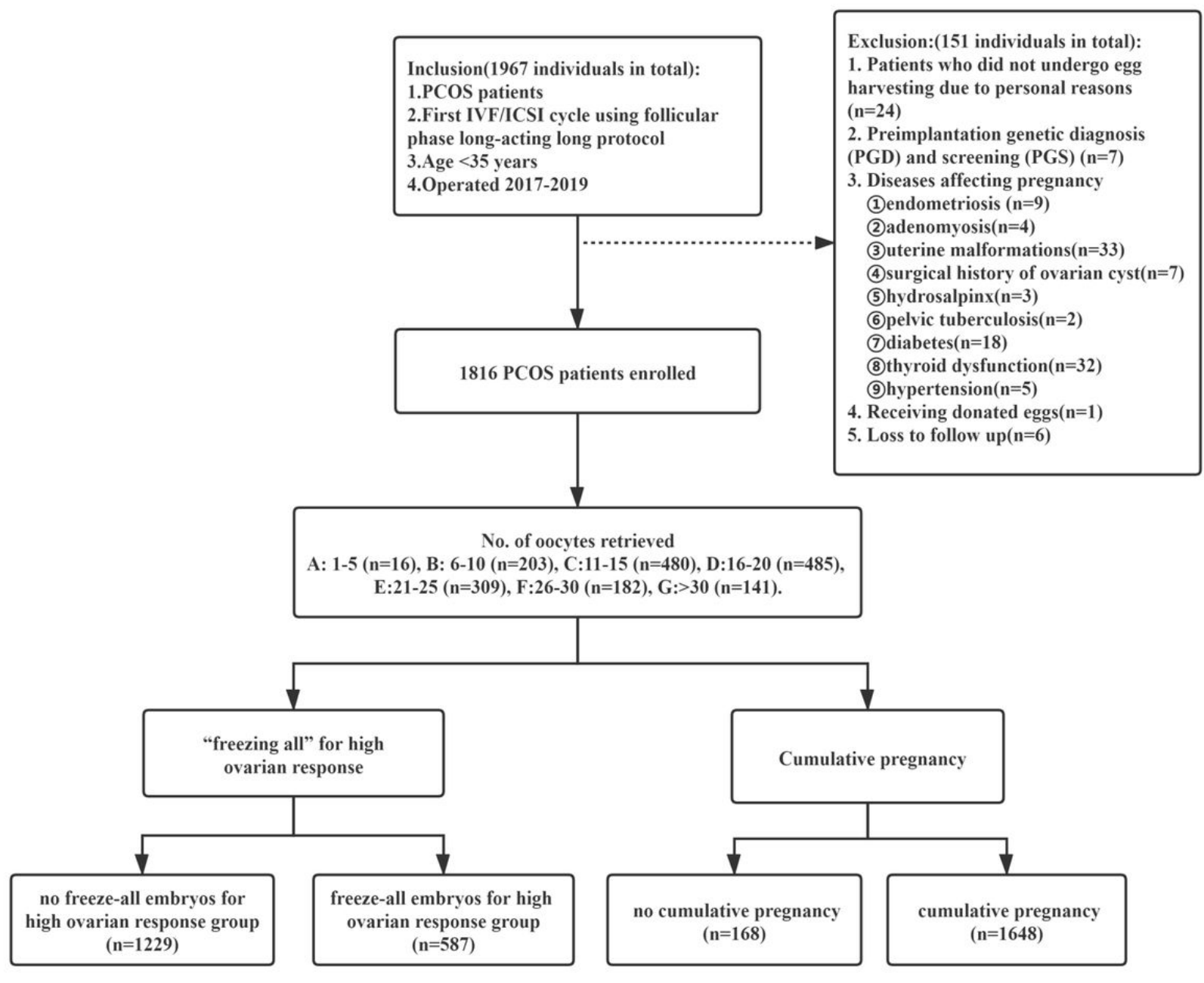

Figure 1

Flow chart depicting the patient selection. 


\section{Distribution of the number of retrieved}

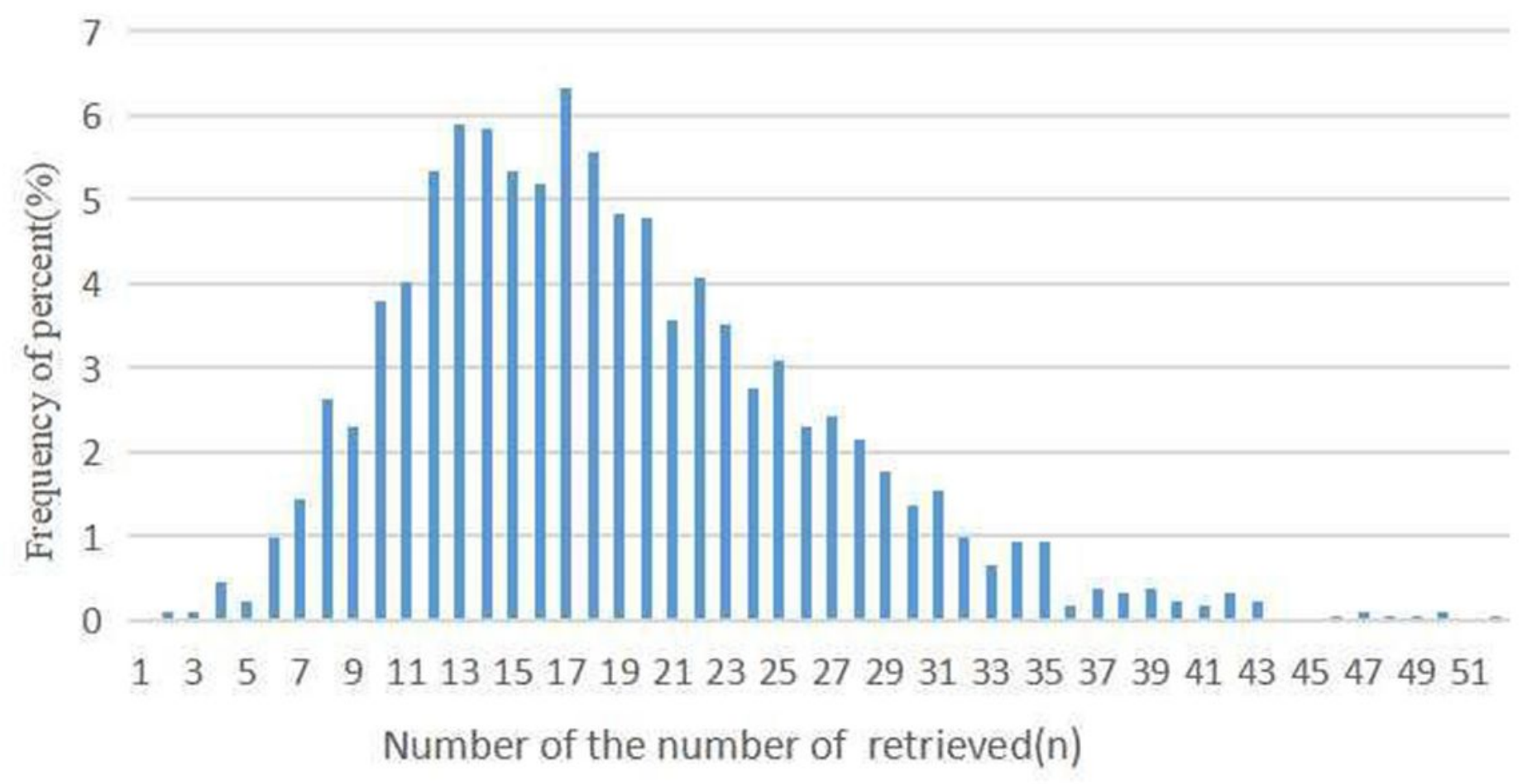

Figure 2

Distribution of the number of oocytes retrieved

Laboratory results and clinical outcomes

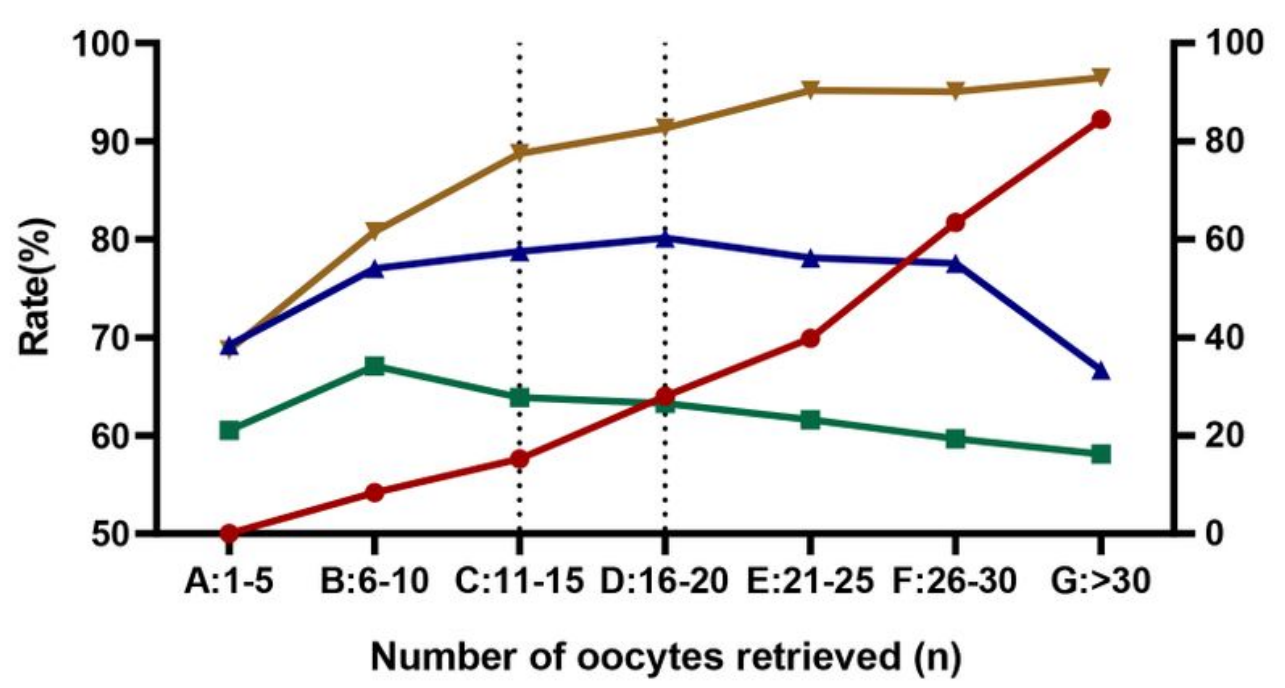
$\rightarrow \quad$ "freezing all" for high

$\rightarrow$ High-quality embryo rate

- Clinical pregnancy rate

$\rightarrow$ Cumulative pregnancy rate

Figure 3

Laboratory results and clinical outcomes Note: High-quality embryo, clinical pregnancy rate, and cumulative pregnancy rate are referring to the ordinate axis on the left; "freezing all" for high ovarian response are referring to the ordinate axis on the right 